\title{
Drawing Area-Proportional Venn and Euler Diagrams
}

\author{
Stirling Chow and Frank Ruskey \\ Department of Computer Science, University of Victoria, Canada \\ \{schow, fruskey\}@cs. uvic.ca
}

\begin{abstract}
We consider the problem of drawing Venn diagrams for which each region's area is proportional to some weight (e.g., population or percentage) assigned to that region. These area-proportional Venn diagrams have an enhanced ability over traditional Venn diagrams to visually convey information about data sets with interacting characteristics. We develop algorithms for drawing area-proportional Venn diagrams for any population distribution over two characteristics using circles and over three characteristics using rectangles and near-rectangular polygons; modifications of these algorithms are then presented for drawing the more general Euler diagrams. We present results concerning which population distributions can be drawn using specific shapes. A program to aid further investigation of area-proportional Venn diagrams is also described.
\end{abstract}

\section{Introduction}

In 1880, John Venn introduced a notation for representing logical propositions using intersecting Jordan curves [1]. These Venn diagrams were specialized instances of a more general notation for representing set relations which Leonard Euler developed in the $18^{\text {th }}$ century [5].

Many people encounter the familiar 2-set and 3-set Venn diagrams shown in Fig. 1 at some point in their mathematical education; they are often used to introduce students to set theory. Informally, an $n$-set Venn diagram is comprised of $n$ Jordan curves The curves divide the plane into $2^{n}$ connected sets of points called regions which uniquely represent all possible intersections of the (open) interiors and exteriors of the curves. For compactness, the intersection symbols $(\cap)$ in Fig. 1 will be omitted from future diagrams.

The utility of Venn diagrams comes by mapping the curve interiors to sets in the problem domain. For example, if curves $A, B$, and $C$ in Fig. 11(b) represent teenagers, smokers, and drinkers, respectively, then region $A B \bar{C}$ would represent teenage smokers who do not drink. If a region is mapped to an empty problem domain set, it is shaded as shown by regions $\overline{A B} C$ and $\bar{A} B C$ in Fig. 2(a).

Euler diagrams are similar to Venn diagrams except they omit shaded regions all-together. Figure[2(b) is an Euler diagram derived from Fig. 2(a) by removing regions $\overline{A B} C$ and $\bar{A} B C$. Euler diagrams are useful because they reduce some of 

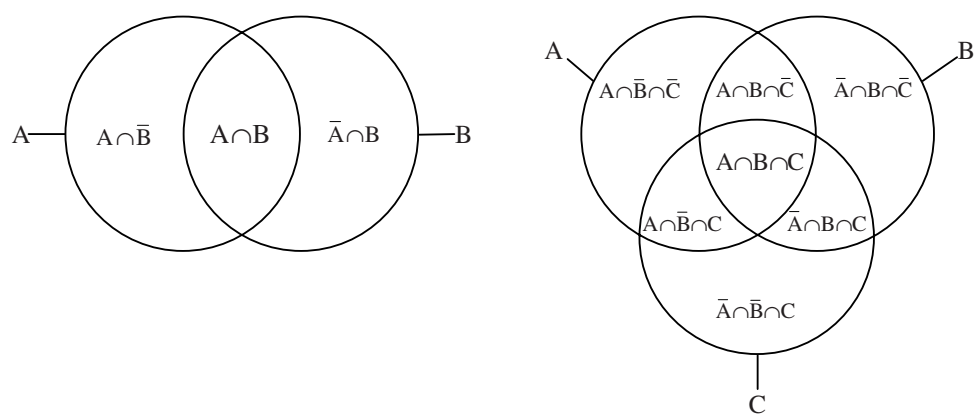

Fig. 1. Common 2-set and 3-set Venn diagrams

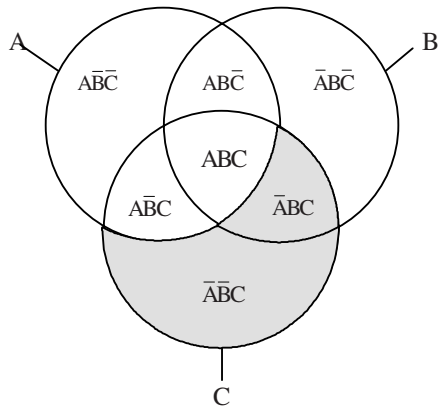

(a)

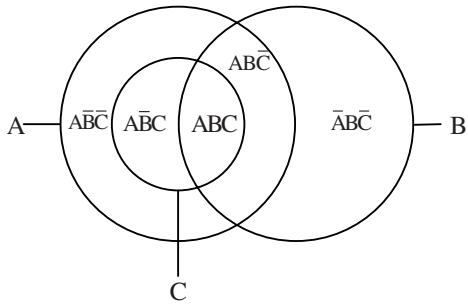

(b)

Fig. 2. A Venn diagram (a) with empty regions and the associated Euler diagram (b)

the clutter associated with Venn diagrams and make certain relationships more evident 8].

In addition to being useful as teaching aids, Venn and Euler diagrams have been used to solve problems in many domains including diagrammatic logic reasoning, finite set counting, and constraint modelling [7]. Irrespective of particular problems, Venn and Euler diagrams can also be used to effectively visualize data sets.

This paper focuses on techniques for drawing Venn and Euler diagrams with an improved ability to convey information about a data set. The work contained herein was inspired after one of the authors published a survey of Venn diagrams [10] and received numerous requests by readers for software to produce diagrams such as the one Fig. 3. (a), but with the added constraint that each region's area should be representative of its associated data. Figure 3 (b) is an example of such an area-proportional Venn diagram.

Although there are algorithms to produce Venn and Euler diagrams for any number of sets [1146], the authors are unaware of any work on drawing algorithms that take area into account. As such, the forthcoming sections will formalize the concept of area-proportional Venn and Euler diagrams, provide 


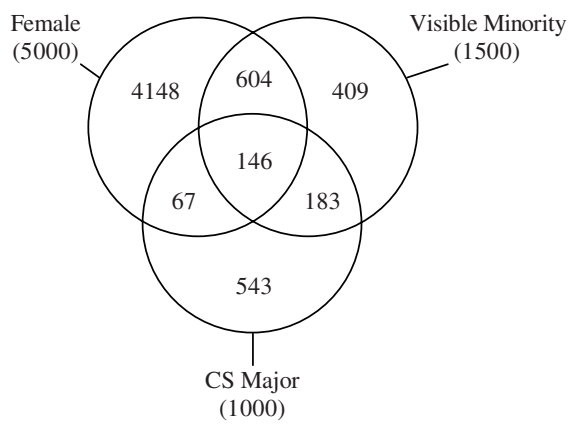

(a)

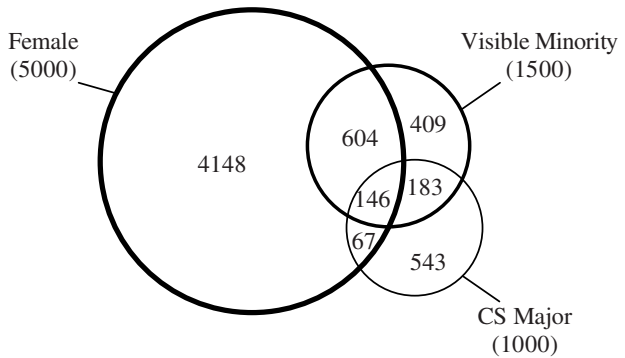

(b)

Fig. 3. A Venn diagram (a) representing a weighted data set and its corresponding area-proportional version (b)

algorithms for drawing 2-set and 3-set Venn and Euler diagrams, and discuss some of the aesthetic qualities desirable in area-proportional diagrams. It is the authors' hope that this paper will provide a solid foundation for future work in the area.

This work is related to graph drawing in the sense that a Venn or Euler diagram can be viewed as a graph embedded in the plane. In the graph drawing literature some attention has been paid to the areas of regions, mainly the aesthetic criteria that the area not get too small in relation to the area of other regions [1].

\section{Formalisms}

Before we proceed with describing the drawing algorithms, we must formally define area-proportional Venn and Euler diagrams. We begin by defining a division of the plane $\mathbb{R}^{2}$ into regions by a collection of Jordan curves.

Definition 1. Let $C=\left\{C_{1}, C_{2}, \ldots, C_{n}\right\}$ be a collection of Jordan curves in $\mathbb{R}^{2}$ and let $\operatorname{int}\left(C_{i}\right)$ and ext $\left(C_{i}\right)$ denote the points of $\mathbb{R}^{2}-C_{i}$ that are interior and exterior, respectively, to $C_{i}$ as defined by the Jordan Curve Theorem [9].

The regions of $C$, denoted $R(C)$, are defined as follows:

$$
R(C)=\left\{X_{1} \cap X_{2} \cap \cdots \cap X_{n} \mid X_{i} \in\left\{\operatorname{int}\left(C_{i}\right), \operatorname{ext}\left(C_{i}\right)\right\}\right\} .
$$

Note that $|R(C)|=2^{n}$. Each region, $X_{1} \cap X_{2} \cap \cdots \cap X_{n}$, is labelled $L_{1} L_{2} \cdots L_{n}$ where $L_{i}=C_{i}$ if $X_{i}=\operatorname{int}\left(C_{i}\right)$ and $L_{i}=\overline{C_{i}}$ if $X_{i}=\operatorname{ext}\left(C_{i}\right)$. For example, region $\operatorname{int}(A) \cap \operatorname{int}(B) \cap \operatorname{ext}(C)$ would be referred to as region $A B \bar{C}$.

Region $\overline{C_{1} C_{2}} \ldots \overline{C_{n}}$ represents the part of the universe exterior to all curves and is designated $U^{\prime}$.

Venn diagrams are a special instance of a collection of curves whose regions satisfy a non-empty and connected condition. 
Definition 2. A collection of curves, $C$, is a Venn diagram if for all $r \in R(C)$, $r$ is a non-empty set of connected points.

For example, Fig. 4 shows two collections of curves that are not Venn diagrams; (a) fails because region $\bar{A} B \bar{C}$ is empty and (b) fails because region $A \bar{B}$ is disconnected.

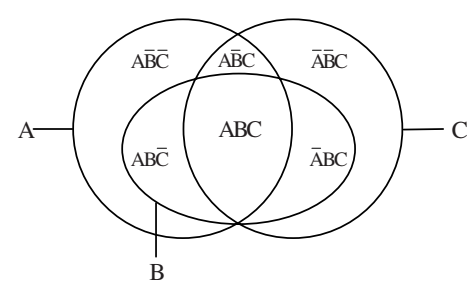

(a)

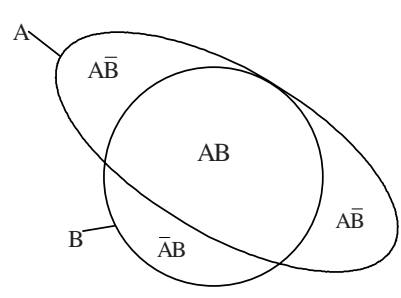

(b)

Fig. 4. Two collections of curves that are not Venn diagrams; however, (a) is an Euler diagram

By relaxing the non-empty constraint on Venn diagrams, we yield a definition for Euler diagrams. Note that because certain regions can be empty, an Euler diagram may have non-intersecting curves.

Definition 3. A collection of curves, $C$, is an Euler diagram if for all $r \in R(C)$, the region $r$ is connected (an empty region is vacuously connected).

For example, returning to Fig. 4. (a) is an Euler diagram, but (b) remains neither a Venn nor an Euler diagram.

The aforementioned definitions of Venn and Euler diagrams are the broadest possible. A number of subclasses of Venn and Euler diagrams have been studied (interested readers should consult [10]), and some researchers define Venn and Euler diagrams at the exclusion of certain subclasses. Although we consider the most general cases of Venn and Euler diagrams, we will discuss the implications of certain characteristics on the diagrams' visual effectiveness. The two characteristics that we will consider are simplicity and finiteness.

Definition 4. A Venn or Euler diagram is simple if no more than two curves intersect at a common point; otherwise, it is termed non-simple.

For example, the Venn diagram in Fig. 1 (b) is simple, but the Euler diagram in Fig. 4(a) is non-simple because of the common intersection of curves $A, B$, and $C$ at the bottom of the diagram.

Definition 5. A Venn or Euler diagram is finite if its curves intersect at finitely many points; otherwise, it is termed infinite. 
All the Venn and Euler diagrams discussed up to this point have been finite. In Section 4 we will encounter infinite Venn diagrams.

Now that we have formally defined Venn and Euler diagrams, we can proceed to formalize the notion of area-proportionality. Area-proportionality only has meaning once problem domain values have been mapped to the regions of a Venn or Euler diagram. The problem domain values may be related to the cardinality of the sets represented by each region as shown in Fig. 3 or they may be other values such as the probabilities shown in Fig. 5. Regardless of their meaning, the values can be treated simply as arbitrary weights assigned to the regions of a Venn or Euler diagram. The idea behind area-proportionality is that a region's area (that is, the smallest area of the plane that encloses the region's points), should be directly proportional to the weight assigned to the region. For example, if region $A$ has a weight twice that of region $B$ then region $A$ should have an area twice that of region $B$.

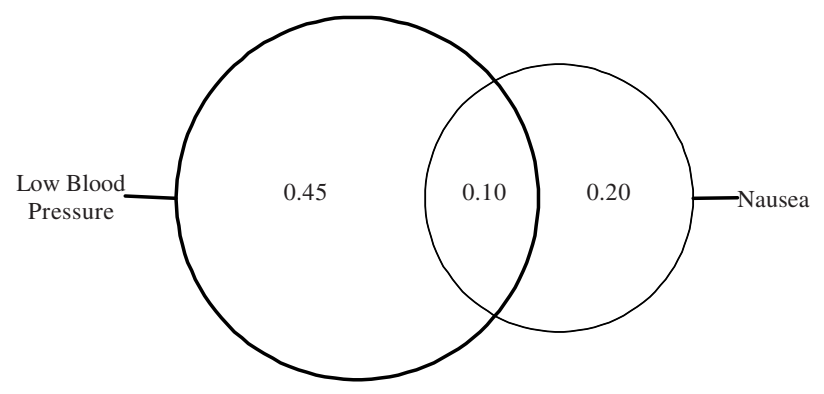

Fig. 5. A area-proportional Venn diagram showing the probabilities of certain complications for a trial drug

Definition 6. Given a Venn or Euler diagram, $C$, and a non-negative function $\omega: R(C) \rightarrow \mathbb{R}$, a diagram $C$ is an area-proportional with respect to $\omega$ if

$$
\frac{\operatorname{Area}(r)}{\sum_{r^{\prime} \in R(C)-U^{\prime}} \operatorname{Area}\left(r^{\prime}\right)}=\frac{\omega(r)}{\sum_{r^{\prime} \in R(C)-U^{\prime}} \omega\left(r^{\prime}\right)} \quad \text { for all } r \in R(C)-U^{\prime} .
$$

Note that since the universe is represented by the unbounded plane, it does not factor into determining the proportions of the diagram. Also, by necessity, a Venn diagram can only be area-proportional to an $\omega$ with positive values.

\section{2-Set Area-Proportional Venn and Euler Diagrams}

Figure [5 shows a 2-set area-proportional Venn diagram with curves that are circles; to compute such a diagram, $G=\{A, B\}$, for a weight function $\omega$, we first calculate the radii of the circles so that their areas equal the sum of their constituent regions' weights: 


$$
r_{1}=\sqrt{\frac{\omega(A \bar{B})+\omega(A B)}{\pi}}, \quad r_{2}=\sqrt{\frac{\omega(\bar{A} B)+\omega(A B)}{\pi}} .
$$

We next choose a canonical orientation for the diagram by centering $A$ in the cartesian plane $\mathbb{R}^{2}$ at $(0,0)$ and centering $B$ at $(d, 0)$ where $d$ is chosen so that the area of overlap between $A$ and $B$ is $\omega(A B)$ (see Fig. 6 ). Note that since $\pi r_{1}^{2}$ and $\pi r_{2}^{2}$ are at least $\omega(A B)$, there is always a $d$ that yields the necessary overlap.

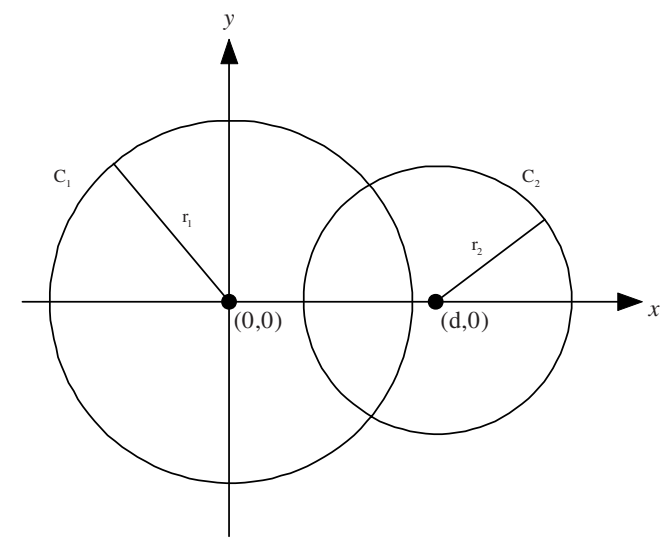

Fig. 6. Canonical form of two-circle diagram

In order to compute $d$, we first need a formula, $\sigma$, for the area of overlap between the two circles; this can be derived by applying Euclidean geometry and trigonometry to yield the following result:

$$
\sigma(d)=\frac{1}{2} r_{1}^{2}(\alpha-\sin (\alpha))+\frac{1}{2} r_{2}^{2}(\beta-\sin (\beta))
$$

where

$$
\alpha=2 \arccos \left(\frac{d^{2}+r_{1}^{2}-r_{2}^{2}}{2 r_{1} d}\right) \quad \beta=2 \arccos \left(\frac{d^{2}+r_{2}^{2}-r_{1}^{2}}{2 r_{2} d}\right)
$$

are in radians.

Because $\sigma$ is not invertible, in order to solve $\sigma(d)=\omega(A B)$ we need to apply numerical methods. Note that $\sigma$ is minimal at $d=r_{1}+r_{2}$, maximal at $d=r_{1}-r_{2}$, and monotonically non-decreasing within this range regardless of the relative sizes of $A$ and $B$ (although if $r_{1}<r_{2}, \sigma$ achieves a maximum at $\left.d=r_{2}-r_{1}\right)$. Because of $\sigma$ 's monotonicity, we can apply a simple bisection algorithm [2] in the range $\left[r_{1}-r_{2}, r_{1}+r_{2}\right]$ to efficiently determine $d$. 
This algorithm produces Euler diagrams without need for any changes. If regions $A \bar{B}$ and $\bar{A} B$ both have weight 0 , the algorithm will produce two equalsized circles centered at $(0,0)$; if only one of these regions has weight 0 , the algorithm will produce a diagram where the circles intersect at a single point and one circle lies within the other. Lastly, if region $A B$ has weight 0 , the algorithm will produce a diagram where the closed interiors of the circles intersect a single point.

The values of $r_{1}, r_{2}$, and $d$ fully specify a $1: 1$ scale diagram with respect to $\omega$. In order to produce a diagram for an output device such as a printer, a standard scaling transformation can be applied. This algorithm provides a constructive proof for the following theorem.

Theorem 1. A 2-set area-proportional Venn or Euler diagram, $G$, whose curves are circles exists with respect to any non-negative weight function $\omega: R(G) \rightarrow \mathbb{R}$.

\section{3-Set Area-Proportional Venn and Euler Diagrams}

It is generally not possible to extend the results of Thm. 10 to three circles; this can be seen by considering Fig. 7. Given a weight function $\omega$, the amount of overlap between circles $A$ and $B$ in Fig. $7(\mathrm{~b})$ is proportional to $\omega(A B \bar{C})+$ $\omega(A B C)$; similarly, for $B$ and $C$ it is proportional to $\omega(A B C)+\omega(\bar{A} B C)$. Using the algorithm from Sec. 3, we can compute the distance between the centers of $A$ and $B$ so that the necessary overlap is achieved; if all the regions are non-empty (i.e., a Venn diagram), then there is only one solution. A similar computation is performed for $B$ and $C$. At this point, we don't know the relative orientation of $A$ and $C$ as shown in Fig. 7(a). To determine the overlap of $A$ and $C$ while maintaining the overlap that has already been established between $A$ and $B$, we revolve $A$ in a fixed-distance orbit about $B$ until region $A B C$ has the proper area as shown in Fig. 7(b); this can be done using polar coordinates and another bisection. At the end of this process, regions $\bar{A} B \bar{C}, A B \bar{C}, A B C$, and $\bar{A} B C$ have the right area, but regions $A \overline{B C}, A \bar{B} C$, and $\overline{A B} C$ may be incorrect. There are no more degrees of freedom in positioning the circles, so in general, there are weight distributions that cannot be represented using three circles.

In order to increase the number of degrees of freedom, more complex curves are necessary. An alternative to circles are ellipses, but the area calculations become unwieldy, and it is unclear how to adjust the ellipse parameters to achieve the desired areas. Because of their amenability to area calculations and discrete manipulation, we will consider how to use axis-aligned rectangles to produce orthogonal drawings [1] of Venn and Euler diagrams. Following is an example to describe an algorithm for producing a 3-set finite Venn diagram, $G=\{A, B, C\}$, given the weight function $\omega$ defined in Tbl. 1

The idea is to build the diagram region-by-region using rectangles whenever possible. We use $W_{r}$ and $H_{r}$ to refer to the width and height, respectively, of region $r$; if $r$ is not rectangular, then $W_{r}$ and $H_{r}$ refer to the longest width and 


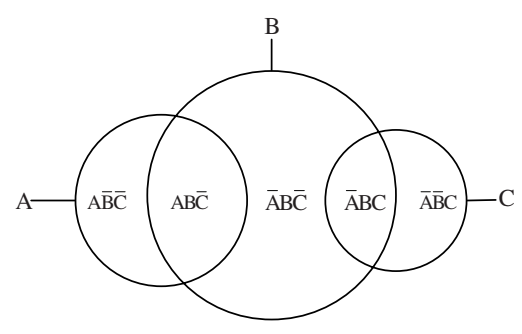

(a)

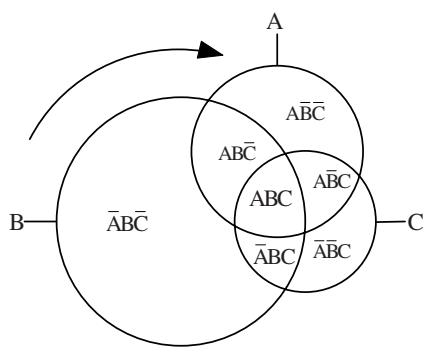

(b)

Fig. 7. A possible algorithm for 3-set area-proportional Venn diagrams

Table 1. Sample weight function

\begin{tabular}{cc}
\hline$r$ & $\omega(r)$ \\
\hline$A B C$ & 2 \\
$A B \bar{C}$ & 6 \\
$A \bar{B} C$ & 6 \\
$\bar{A} B C$ & 1 \\
$\bar{A} \overline{B C}$ & 3.5 \\
$\overline{A B} C$ & 7 \\
$\bar{A} B \bar{C}$ & 9 \\
\hline
\end{tabular}

height. The first step is to choose a value for $W_{A B C}$ and place the resulting rectangle on the plane. In this example we choose $W_{A B C}=2$ and place a $2 \times 1$ rectangle on the plane.

Next, we place a $W_{A B C} \times \frac{\omega(A \bar{B} C)}{W_{A B C}}$ rectangle above $A B C$ for $A \bar{B} C$, and similarly a $\frac{\omega(\bar{A} B C)}{H_{A B C}} \times H_{A B C}$ rectangle to the right of $A B C$ for $\bar{A} B C$.

For $A B \bar{C}$ we need to decide how much of it to place below $A B C$ and how much of it to place to the left of $A B C$. We let $d$ be the vertical distance of $A B \bar{C}$ below $A B C$, and we can choose any value for this as long as $W_{A B C} \cdot d<\omega(A B \bar{C})$. Once $d$ is chosen, $A B \bar{C}$ will be the union of a $\frac{\omega(A B \bar{C})-W_{A B C} \cdot d}{H_{A B C}+d} \times\left(H_{A B C}+d\right)$ rectangle and a $W_{A B C} \times d$ rectangle. In this example we choose $d=1$ and the resulting diagram is shown in Fig. 8 (a).

Now that the core of the diagram has been established, the remaining regions are filled in as best as possible to create rectangular curves. Referring to Fig. \& $(\mathrm{b})$, if $\omega(\overline{A B} C)>W_{\bar{A} B C} \cdot H_{A \bar{B} C}$, then $\overline{A B} C$ can fill the corner between $\bar{A} B C$ and $A \bar{B} C$ before overflowing to the right of $\bar{A} B C$. Similarly, if $\omega(\bar{A} B \bar{C})>d \cdot W_{\bar{A} B C}$, then $\bar{A} B \bar{C}$ can fill the corner between $A B \bar{C}$ and $\bar{A} B C$ before overflowing below and to the left of $A B \bar{C}$.

In the case of $A \overline{B C}, \omega(A \overline{B C}) \leq W_{A B \bar{C}} \cdot H_{A \bar{B} C}$ so the corner between $A B \bar{C}$ and $A \bar{B} C$ cannot be filled to create a rectangular curve $A$. As a result, we 


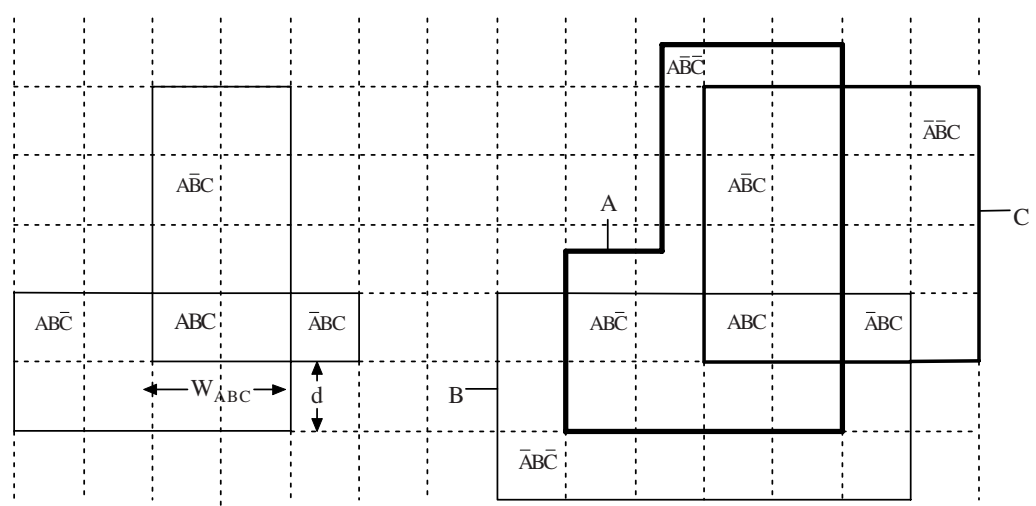

(a)

(b)

Fig. 8. Example of 3-set infinite area-proportional Venn diagram algorithm

evenly distribute $A \overline{B C}$ around the top of $A B \bar{C}$ and top left corner of $A \bar{B} C$. An analogous rule would hold for $\overline{A B} C$ and $\bar{A} B \bar{C}$ where they not sufficiently large to fill there associated corners.

There are a few things to note about this algorithm. First, if a curve cannot be made rectangular, then its shape is that of a rectangle with a corner cut out (see curve $A$ in Fig. $8($ (b)); we call these shapes near-rectangular. Second, the choice to overflow $\overline{A B} C$ to the right of $\bar{A} B C$ is arbitrary. $\overline{A B} C$ could overflow on top of $A \bar{B} C$ instead which would have caused the subsequent regions to "rotate" counterclockwise 90 degrees, and although this results in a different diagram, the criteria concerning whether or not the resultant curves are rectangular does not change. Third, once $W_{A B C}$ and $d$ are chosen, the rest of the diagram is determined (according to the algorithm). Fourth, the choice of $d$ can effect whether or not the resulting curves are rectangular. Larger values of $d$ decrease the width of $A B \bar{C}$ to the left of $A B C$ which might allow $A \overline{B C}$ to fill its corner and create a rectangular curve $A$; however, there is a trade-off since larger values of $d$ create a larger corner for $\bar{A} B \bar{C}$ to fill and may result in curve $B$ becoming near-rectangular.

The last point is important because it suggests that the choice of $d$ is critical to achieving a diagram comprised of rectangles. The following theorem is a direct result of this observation.

Theorem 2. A simple 3-set area-proportional Venn diagram, $G=\{A, B, C\}$, whose curves are axis-aligned rectangles exists with respect to a positive weight function $\omega: R(G) \rightarrow \mathbb{R}$ if, and only if, there exists a permutation, $\pi$, of $\{A, B, C\}$ such that $\Omega(r)=\omega(\pi(r))$ (that is, $\Omega$ permutes the regions' labels) and the following conditions hold:

$$
\max \left(0, \frac{\Omega(A B \bar{C}) \cdot \Omega(A \bar{B} C)-\Omega(A \overline{B C}) \cdot \Omega(A B C)}{\Omega(A \overline{B C})+\Omega(A \bar{B} C)}\right)
$$




$$
<\min \left(\Omega(A B \bar{C}), \frac{\Omega(\bar{A} B \bar{C}) \cdot \Omega(A B C)}{\Omega(\bar{A} B C)}\right)
$$

and

$$
\frac{\Omega(\bar{A} B C) \cdot \Omega(A \bar{B} C)}{\Omega(A B C)}<\Omega(\overline{A B} C) .
$$

Proof. For the sake of space, we provide only a sketch of the proof. For sufficiency, we establish the inequalities that must be satisfied in order for all curves to be rectangular; that is, we require the corners of Fig. 8(a) to be small enough to permit filling by the subsequent regions. By simultaneously solving these inequalities, we derive the conditions of the theorem. The addition of $\Omega$ is to allow for all possible orderings of the curves around the diagram.

For necessity, we consider a three rectangle Venn diagram and remove one of the curves. The resulting diagram has two intersecting curves whose shared region is itself a rectangle. In order to be a Venn diagram, the third curve must bisect each region exactly once which results in a core that is isomorphic to Fig. 8 (a) under rotation and reflection. The result then follows.

Even if a rectangular Venn diagram cannot be produced, the resulting curves will at least be near-rectangular. In addition, the algorithm can also be applied without change for Euler diagrams. As long as $\omega(A B C)>0$, the algorithm functions correctly; however, it is not optimal in the sense that some infinite Euler diagrams are produced that could be finite. The case when $\omega(A B C)=0$, remains an open problem.

\section{Conclusion}

In order to experiment with various drawing algorithms and to better understand some of the user interface issues concerning area-proportional diagrams, we have developed a Java application called DrawVenn that implements the 2-set and 3 -set Venn and Euler algorithms described in this paper. As shown in Fig. 9, a DrawVenn user can dynamically adjust the weight function and view the updated diagram. There is also a control for adjusting algorithm-specific parameters such as the values of $W_{A B C}$ and $d$ in the 3-set algorithm. Within DrawVenn, the curves are filled with user-selectable transparent colors that are alpha-blended to provide an intuitive cue as to which curves are intersecting in a given region. DrawVenn can also output color bitmaps as well as scalable vector formats. In the case of vector formats, DrawVenn produces monochrome drawings and varies the thickness of the curves to indicate their relative sizes.

We are also interested in understanding which aesthetic characteristics of an area-proportional diagram affect its ability to convey information. A person viewing an area-proportional Venn or Euler diagram needs to be able to determine which curves enclose a region (i.e., what the region represents) and how the size of a region compares to other regions. 


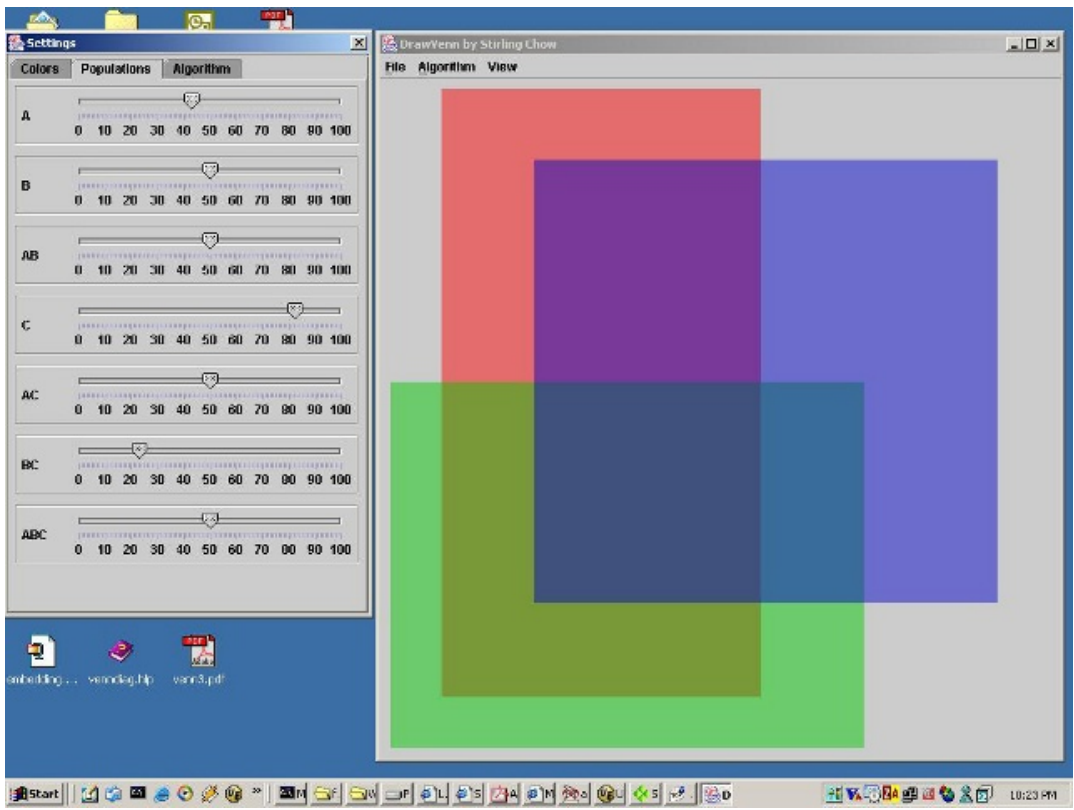

Fig. 9. Screenshot of DrawVenn showing sliders for dynamic adjustment of populations

In order to determine a region's enclosing curves, the curves must be distinguishable and easy to trace (i.e., follow with the eye). On the surface, it would seem that the curves of an infinite Venn or Euler diagram are less distinguishable than the curves of a finite one. Similarly, it would seem that the curves of a non-simple Venn or Euler diagram are more difficult to trace than the curves of a simple diagram since there are more options for where a curve exits a vertex. According to the gestalt "good continuation" principle [3], smooth curves should also be more traceable than ones whose direction changes sharply, thus providing some motivation for developing a 3-set algorithm that uses ellipses rather than rectangles.

In order to compare regions, their shapes should be similar. For example, it is easier to compare the relative size of a circle to another circle than it is to a triangle. The orthogonal diagrams hold an advantage in this area since their regions are largely rectangular; however, the areas of rectangles can be difficult to compare if their aspect ratios are vastly different. The measure of a diagram's regional uniformity may be a good indicator of its effectiveness.

Area-proportional Venn and Euler diagrams have an advantage over traditional diagrams because they leverage both an individual's perceptual and cognitive abilities. We hope that the algorithms described in this paper provide a first step in the direction of understanding how best to utilize area-proportional diagrams, and we hope others will share our interest in this area. 


\section{References}

[1] G.D. Battista, P. Eades, R. Tomassia, and I.G. Tollis. Graph Drawing: Algorithms for the Visualization of Graphs. Prentice-Hall, 1999.

[2] Richard L. Burden and J. Douglas Faires. Numerical Analysis: 4th Edition. PWS Publishing Co., 1988.

[3] Stuart K. Card, Jock D. Mackinlay, and Ben Shneiderman. Readings in Information Visualization: Using Vision to Think. Morgan Kaufmann Publishers, 1999.

[4] Anthony W.F. Edwards. Venn diagrams for many sets. New Scientist, 7:51-56, January 1989.

[5] Leonard Euler. Lettres a Une Princesse d'Allemagne, volume 2. 1761. Letters no. $102-108$.

[6] Jean Flower and John Howse. Generating Euler diagrams. In Proceedings of Diagrams 2002, pages 61-75. Springer-Verlag, April 2002.

[7] Joseph (Yossi) Gil, Stuart Kent, and John Howse. Formalizing spider diagrams. In Proceedings of the IEEE Symposium on Visual Languages, pages 130-137. IEEE Computer Society Press, September 1999.

[8] Joseph (Yossi) Gil, Stuart Kent, John Howse, and John Taylor. Projections in Venn-Euler diagrams. In Proceedings of the IEEE Symposium on Visual Languages, pages 119-126. IEEE Computer Society Press, September 2000.

[9] Konrad Knopp. Theory of Functions, Parts I and II, Two Volumes Bound as One, volume 1. Dover, 1996.

[10] Frank Ruskey. A survey of Venn diagrams. Electronic Journal of Combinatorics, 4, 1997 (update 2001). DS\#5.

[11] John Venn. On the diagrammatic and mechanical representation of propositions and reasonings. The London, Edinburgh, and Dublin Philosophical Magazine and Journal of Science, 9:1-18, 1880. 